\title{
COVID-19: Causes of anxiety and wellbeing support needs of healthcare professionals in the UK: A cross-sectional survey
}

\author{
Authors: Imrana Siddiqui, ${ }^{A}$ Marco Aurelio, ${ }^{B}$ Ajay Gupta, ${ }^{C}$ Jenny Blythe ${ }^{D}$ and Mohammed $Y$ Khanji ${ }^{E}$
}

\section{Background}

COVID-19 has caused acute changes in healthcare delivery; this may impact mental health and wellbeing needs of healthcare professionals (HCPs).

Aims

We aimed to identify the causes of anxiety in HCPs during the COVID-19 pandemic, to assess whether HCPs felt they had adequate mental health and wellbeing support and to identify their unmet support needs.

\section{Method}

We used a web-based survey utilising an online tool circulated to UK HCPs over 5 weeks. Self-perceived anxiety levels prior to and during the COVID-19 pandemic were measured on a 10-point Likert-type rating scale.

Results

The survey was completed by 558 HCPs. During the pandemic, self-perceived anxiety scores significantly increased from a median of 2 to 7 (paired Wilcoxon signed-rank test; $p<0.001$ ). The main reasons were concerns about exposure to SARSCoV-2 and lack of personal protective equipment. Other wide-ranging reasons were identified. Only $41 \%$ of respondents felt there was adequate support. Thematic analysis of what support HCPs wanted identified 13 wide-ranging themes; including effective leadership and peer support.

Conclusion

Anxiety levels in HCPs significantly increased during the COVID-19 pandemic and the main causes were identified.

Authors: ${ }^{\text {A }}$ general practitioner, Woodgrange Medical Practice, London, UK, Wellbeing Hub lead, Newham Training Hub, London, UK and clinical lead for mental health and learning disabilities, Waltham Forest and East London clinical commissioning groups,

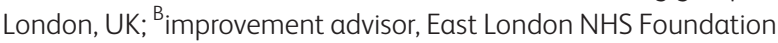
Trust, London, UK; ' Consultant in clinical pharmacology and cardiovascular medicine, Barts Health NHS Trust, London, UK and senior clinical lecture, Barts Cardiovascular Clinical Trials Unit, London, UK; ' general practitioner, Liberty Bridge Road Practice, London, UK and senior clinical lecturer, Queen Mary University of

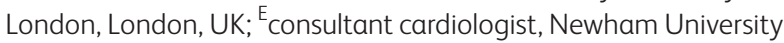
Hospital, London, UK and honorary senior clinical lecturer, William Harvey Research Institute, London, UK
Many HCPs felt there was inadequate support and identified what support they needed. Implementing effective strategies to support HCPs' unmet wellbeing needs are required as a matter of urgency.

KEYWORDS: COVID-19, anxiety, healthcare professionals, mental health, wellbeing

DOI: $10.7861 /$ clinmed.2020-0502

\section{Introduction}

The COVID-19 pandemic is one of the most significant world health crises of this generation. The impact on the lives of people worldwide is unprecedented. The Office for National Statistics in the UK reported a sharp rise in the level of anxiety in the adult population during the COVID-19 pandemic; $49.6 \%$ of people reported 'high' (rating 6-10) anxiety during pandemic compared with $21 \%$ at the end of $2019 .{ }^{1}$ A systematic review and metaanalysis showed the prevalence of anxiety as $31.9 \%$ and of depression as $33.7 \%$ among the general population during the COVID-19 pandemic. $^{2}$

In particular, looking at healthcare professional (HCPs); a survey from China during the COVID-19 pandemic of more than 1,200 HCPs found that about 50\% reported at least mild depression; with over $10 \%$ of physicians and nurses reporting moderate or severe depressive symptoms. ${ }^{3}$ A US study reported that HCPs are at greater risk than the general public of experiencing mental health problems, such as depression, during the COVID-19 pandemic. $^{4}$

Other survey-based studies in the UK exploring the psychological impact of the COVID-19 pandemic on HCPs are currently underway. The CERA study is looking at the impact on physicians working in the emergency care setting and the COPE-HCP study looks at HCPs internationally alongside a non-HCP control arm., 5

A rapid review of the psychological impact of an epidemic/ pandemic on the mental health of HCPs looking at all epidemics/ pandemics since 2003 (including severe acute respiratory syndrome (SARS), Middle East respiratory syndrome (MERS), H1N1 influenza, avian (H5N1) influenza and West Nile fever) showed that exposed HCPs are at heightened risk of mental health problems in the short and longer term, particularly psychological distress, insomnia, alcohol/drug misuse, and symptoms of post-traumatic stress disorder (PTSD), depression, anxiety, burnout, anger and 
higher perceived stress. ${ }^{7}$ They and others highlight the lack of evidence-based early interventions in this area. ${ }^{8}$ HCPs are less likely to engage in 'proactive coping', meaning they were doing less to prepare themselves for future stresses or adverse events; further highlighting the need for effective interventions. ${ }^{9}$

We hypothesised that the anxiety levels of HCPs in the UK would be increased during the COVID-19 pandemic and wanted to understand the specific reasons for it. We also wanted to explore what mental health and wellbeing support HCPs felt that they needed.

\section{Method}

A cross-sectional survey was undertaken using the SurveyMonkey platform and circulated via email and social media platforms. The survey was conducted over a period of 5 weeks during the height of the first peak of the COVID-19 pandemic in the UK; from 30 March 2020 to 05 May 2020. Considering the pressures facing HCPs, the survey was designed to be as short as possible. No personal identifiable data were collected.

HCPs were asked to self-rate their anxiety levels on a 10-point Likert-type rating scale before and during the COVID-19 pandemic. They were also asked to select the three most likely reasons for any change in anxiety levels. This was measured using a combination of multiple-choice questions with a list of 10 defined answers and an option for free text responses. Respondents were asked if they felt that there were adequate mental health and wellbeing support services, and this was measured using a three point 'yes, no and don't know' scale. The types of support HCPs felt would be most useful were measured using an open-ended question with free text responses. Demographic data were collected including age range, sex, job role, sector and location of work (see supplementary material S1 for full survey).

\section{Statistical analysis}

Ordinal data were expressed as mean ( \pm standard deviation) if normally distributed, otherwise as median and interquartile range. Paired Wilcoxon signed-rank test was used to assess change in anxiety before and during the COVID-19 pandemic in individuals due to the data being ordinal. Free text responses were analysed using thematic analysis (TA). A reflective six-phase TA method was used with an inductive approach.

Institutional approval was obtained through the East London NHS Foundation Trust Mental Health Transformation Programme as part of a wider quality improvement project. Ethics approval was not required given the type of project.

\section{Results}

\section{Demographics}

A total of 558 HCPs answered the survey. Average survey completion time was 2 minutes. Participants were from a variety of HCP groups, with the largest group being doctors (51\%). Of the doctors, general practitioners (GPs) made up 33\%, hospital doctors $16 \%$, community doctors $1.6 \%$ and GP trainees $1 \%$ (Fig 1). Nurses made up $13 \%$ of respondents and were from a variety of health settings: $21 \%$ from primary care, $37 \%$ in hospital settings, $36 \%$ from community settings and $6 \%$ from other settings. Other HCPs who completed the survey included pharmacists, midwives, psychologists, support workers, optometrists, dentists and others.

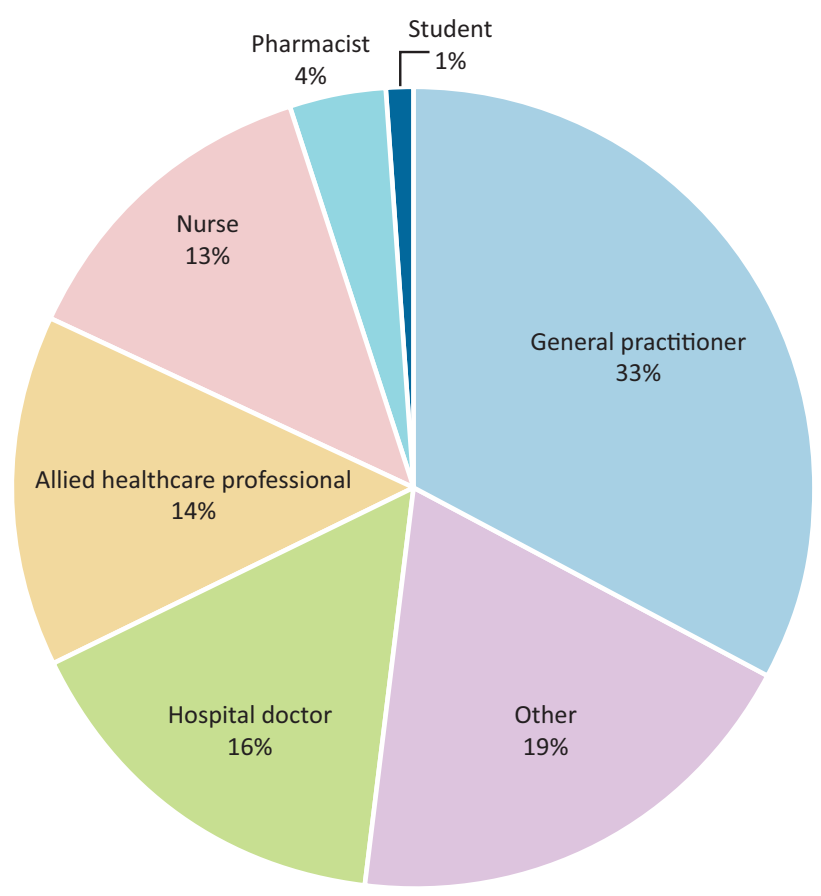

Fig 1. Survey respondents' job roles.

Various healthcare sectors were represented with $39.7 \%$ working in primary care (defined as within general practice surgeries), $26.4 \%$ in hospitals, $25.7 \%$ in the community and $8.3 \%$ in other sectors. Seventy-two per cent of respondents worked in London, with $60 \%$ of them from northeast London, with the remaining $28 \%$ distributed across other parts of England, Scotland, Wales and Northern Ireland.

The majority of the respondents ( $>94 \%$ ) were aged between $25-65$ years old with $3.8 \%$ aged $18-24$ years old and $1.7 \%$ aged $65-74$ years old. Females accounted for $77 \%$ of the respondents.

\section{Anxiety levels and causes}

The self-rated anxiety score increased significantly during the COVID-19 pandemic compared with pre-pandemic level. The median anxiety score rose from 2 to 7 ( $<<0.001$; Fig 2). Breakdown by job role revealed pre-pandemic anxiety levels were very similar across all job roles with a median of 2 . However, during the COVID-19 pandemic they increased, with the highest increase seen in doctors (GPs and hospital) and nurses (supplementary material S2, Fig S1). A median anxiety score of 7 in doctors and nurses compared with 5 and 6 in other professions. Looking at the nurses' data by sector of work revealed the highest anxiety score was in nurses working in hospitals. Their pre-pandemic median anxiety score was 2 and, during the COVID-19 pandemic, this increased to a median score of 10 . However, median anxiety score was lower for primary care and community settings; both having a median anxiety score of 1 pre-pandemic and 7 during the pandemic.

Anxiety levels, by sector of work, showed the biggest increases in primary and secondary care compared with community and 'other' (Fig 3). Prior to the COVID-19 pandemic, median anxiety score of 2 was reported by HCPs from all sectors. During 


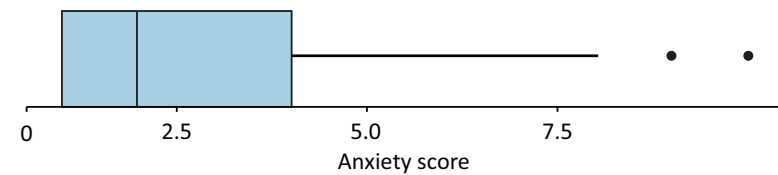

b

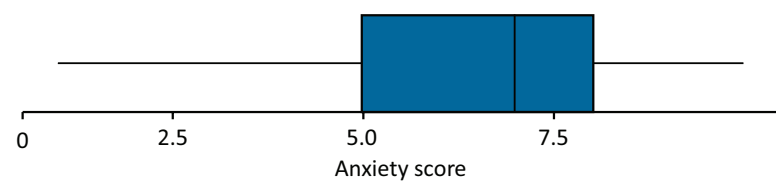

Fig 2. Level of anxiety in healthcare professionals. a) Anxiety prior to the COVID-19 pandemic. b) Anxiety during the COVID-19 pandemic.

the COVID-19 pandemic, this increased to a median of 6 in community and other sectors and increased to 7 in primary care and hospital sectors.

The factors identified as making them most anxious were mainly related to risk of exposure to SARS-CoV-2 to themselves, family and patients, and related to lack of testing and lack of personal protective equipment (PPE). A wide variety of other reasons were also given (Fig 4).

Comparing sectors of work, more HCPs in primary care and hospital settings put lack of PPE, lack of testing and risk of exposure to SARS-CoV-2 as the main causes of anxiety compared with community and 'other' settings (supplementary material, Fig S2). Sixty-six per cent of HCPs working in primary care stated lack of PPE as one of their top three causes of anxiety, compared with $61 \%$ in hospital, $43 \%$ in community and $31 \%$ in 'other' sectors.

Thematic analysis was conducted on the 68 free-text responses for other reasons causing anxiety. Several were applicable in the listed categories and were manually added to those. Other themes are outlined in Table 1. Nine main themes were generated with a wide variety of factors including transferred anxiety from colleagues and patients, limited remote working options, new clinical uncertainties specific to the pandemic, and concerns about the impact of reduced usual healthcare service provision on patients.

a

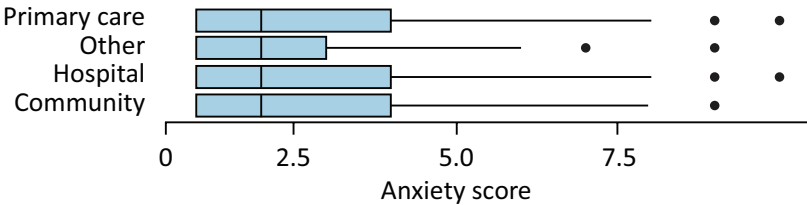

b

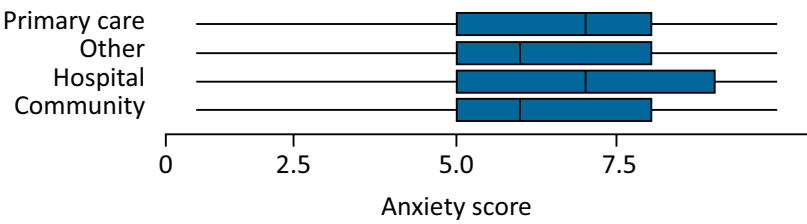

Fig 3. Level of anxiety by work sector. a) Anxiety prior to the COVID-19 pandemic. b) Anxiety during the COVID-19 pandemic.

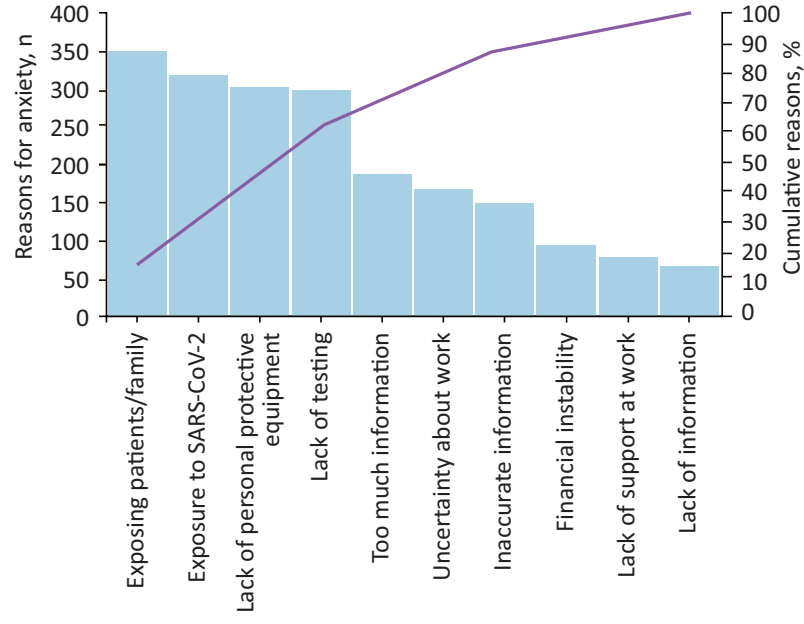

Fig 4. Main reasons for anxiety in healthcare professionals. The blue bars are for individual reasons, the purple line is for the cumulative percentage of reasons.

\section{Mental health and wellbeing support}

Forty-two per cent of total respondents felt that there were adequate resources and services available to support their mental health and wellbeing during the pandemic, $32 \%$ felt that there was not and $28 \%$ responded as 'don't know'.

In the GP subgroup, the responses were more evenly split: 33\% felt there was adequate support, 34\% felt there was not and 33\% responded as 'don't know'. Hospital doctors as a group had the highest feeling of having inadequate support (38\%).

In the nurse subgroup, respondents who felt that there was adequate wellbeing support varied greatly between sectors of work: $20 \%$ in primary care, $31 \%$ in hospital settings, $52 \%$ in community and $25 \%$ in other settings.

Comparing sector of work, $30 \%$ of respondents from primary care and $38 \%$ of respondents from hospital settings, felt they had adequate support compared with $55 \%$ in the community and $55 \%$ in other sectors (Fig 5).

Those who answered 'no' or 'don't know' were asked to comment on what resources and services they would like offered. There were 125 free text comments which were analysed using TA. Thirteen wide-ranging themes were generated from this: workplace-based support, clearer signposting, peer support, wellbeing activities, psychological support and therapies, staff helpline/telephone support, cultural/faith-based support, adequate PPE, support around specific clinical issues related to COVID-19, effective leadership and communication, bereavement support, and tailored support (Table 2).

\section{Discussion}

\section{Anxiety levels and causes}

Anxiety levels were significantly increased in all HCPs roles and sectors during the COVID-19 pandemic. The pre-pandemic anxiety levels were similar in all the HCP roles, but increased most in nurses, doctors and in those working in primary care and hospital settings. The main causes of anxiety were related to 
Table 1. Thematic analysis summary of main reasons for anxiety

\section{Causes of anxiety (themes generated)}

Fear of dying and leaving dependents

Transferred anxiety

Lack of remote working options

Reduced usual service provision

Clinical uncertainty

General uncertainty

Social impact of COVID-19

Change in work setting

Leadership issues

\section{Comments}

Concerns about leaving dependents, in particular, when both parents were key workers and leaving children orphaned, and fear of loved ones dying.

Anxiety transferred from colleagues, managers, employees and patients, and consequent work-based conflicts.

Inability to work from home or to work from home effectively due to IT infrastructure, resources and support issues.

Concerns about adverse impact on patients due to reduced services and routine care; staff sickness; and reduced usual support networks.

Issues around working safely in non-face-to-face consultations, managing risk without examining patients, and how to manage patient anxiety.

Concerns about impact on economy, length of pandemic, and people not following lockdown rules.

Concerns about social isolation, reduced usual support networks, childcare issues, and panic buying.

Problems with increased workload, cancelled leave, and changing landscape (specifically locum general practitioners): feeling unsupported, reduced demand for work and lack of locum options to work remotely.

Lack of trust in national leadership and lack of government preparedness were mentioned. Lack of coordination creating confusion and increased workload eg how to identify and manage high-risk patients. feeling at risk from exposure to SARS-CoV-2. Doctors and nurses often have direct patient facing roles, and therefore may have specific concerns about lack of PPE and exposure to SARS-CoV-2; a likely explanation for their higher levels of anxiety. Although the numbers were small, the most dramatic result was the median anxiety score of 10 (the highest score on the scale) in hospital nursing staff. These nurses, as well as having patient facing roles, are likely to have the longest duration of contact with patients, which increases the risk from exposure to SARS-CoV-2.

General issues around frequent changing guidelines, lack of information, poor communication and training needs were identified. As well as the perceived lack of PPE, other issues such as changing guidelines around PPE, education around the efficacy of different PPE levels and training in using PPE may impact on HCPs anxiety. Interestingly, respondents from hospital settings and primary care both had high levels of concern about inadequate PPE despite individual general practice surgeries in primary care, to an extent, having more control over their own PPE. Safety and security is one of the basic needs from Maslow's hierarchy of needs. ${ }^{10}$ Perceptions of lack of PPE and increased risk of exposure

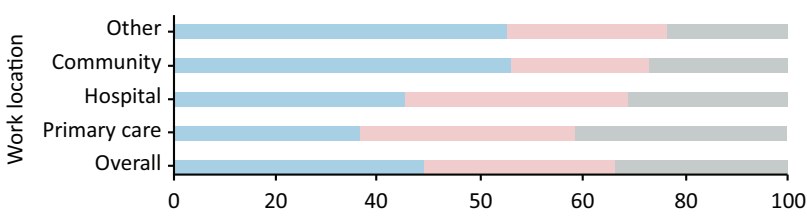

Response to the question 'Do you feel there are adequate support services', \% Yes No Don't know

Fig 5. How healthcare professionals feel about adequate support services by job sector. to SARS-CoV-2 may have a negative effect on the feeling of safety. When trying to address mental health and wellbeing of staff, these basic needs should be actively addressed.

Issues with information were highlighted as a concern; HCPs felt there was either too little or too much information and that it was inaccurate. This has been seen in other pandemics; social media was specifically mentioned as a cause of anxiety, in particular, for its role in spreading inaccurate information during the Zika virus pandemic. $^{11}$

Other causes of anxiety ranged from general global and national uncertainty and specific issues within the workplace and home.

\section{Mental health and wellbeing support}

Many HCPs felt there was inadequate support for their mental health and wellbeing. More HCPs working in primary care and hospital settings felt there was inadequate support than in the community and 'other' sectors. It would be useful to explore the differences among the support services in these different healthcare settings and share good practice and learning. Interestingly, although nurses in the hospital setting felt more anxious, it was nurses in primary care who felt least supported, with only $20 \%$ saying they felt that there was adequate support. A meta-analysis looking at risk factors for psychological distress in HCPs and successful measures to manage them during previous pandemics found that psychosocial and workplace measures can improve employees' working lives and suggests that these, in turn, can improve mental wellbeing even though they are not explicitly mental health support services. ${ }^{12}$ Self-care is also critical given the benefits of diet, exercise and sleep. ${ }^{13}$ Clear communication, access to adequate PPE, adequate rest, and both practical and psychological support were associated with better psychological outcomes. $^{12}$ 
Table 2. Thematic analysis summary of what support healthcare professionals want

What support would healthcare professionals like

Workplace-based support

\section{Clearer signposting}

Peer support

Psychological support and therapies

Staff helpline/telephone support

Wellbeing activities

Support around specific clinical issues related to COVID-19

\section{Cultural/faith-based support \\ Bereavement support}

Effective leadership and communication

\section{Adequate/more personal protective equipment}

Support from friends and family

Tailored support

\section{Comments}

Holistic support for mental health by employers, direct communication from senior staff, support regarding their role, onsite wellbeing support and choice to work from home, supportive visible managers and seniors, and more supervision.

Information and advertising about what support and resources are available and a single point of COVID-19-related information.

Balint groups, safe spaces, multidisciplinary team meetings and one-to-one debrief sessions, talking to colleagues and webinars, and protected time for peer support

Guided self-help, talking therapies, services which are free, short waiting lists for psychological support, and help that is specific for healthcare professionals and specific to COVID-19.

Dedicated phone line for frontline staff, and direct telephone or video line tailored to COVID-19 issues.

Mindfulness, yoga, fitness, physical activities, massage and relaxation sessions.

How to discuss do not attempt resuscitation and ceiling of care, support managing COVID-19 illness, feeling involved in updates rather than just being emailed information.

Cultural approach and faith-based support options.

Specific to not being able to visit dying relative and attending funerals.

From the government and from others in leadership roles; proactive, truthful leadership; and better coordination.

Easy and inexpensive access to personal protective equipment, better protection for face-to-face contact.

Some felt that support from friends and family was sufficient for supporting their mental health and wellbeing.

Tailored, sensitive, local support.
Having adequate resources to support the mental health and wellbeing of HCPs is important after the pandemic as well as during it, as previous studies have highlighted the medium and long-term psychological impact of such events. ${ }^{14}$

As we appear to have passed the first peak of the pandemic in the UK at the time of writing, it would be useful to assess whether anxiety returns to baseline levels and whether over time more HCPs feel adequate support was made available. At the time of the survey, a number of support mechanisms and resources were already being offered, including Practitioner Health Programme, Good Thinking digital mental health resources, and various wellbeing apps being offered free for NHS staff. ${ }^{15-17}$ Various toolkits are also being developed to support HCPs wellbeing including Psychological Wellbeing for Health and Care Workers toolkit developed by Nottingham University, and the COVID-AAD study is looking at the effectiveness of using an adversity reflection tool. $^{18,19}$

However, it appears that many HCPs were not aware of them. This highlights the need for effective and streamlined communication to ensure awareness of what is available. Many professional societies and NHS services have identified and attempted to address some of these needs, however, their usefulness, uptake and effectiveness should be assessed.
NHS Employers commissioned a report evaluating health and wellbeing interventions for healthcare staff. Some of their key findings were that although the trusts involved appreciated the importance of evaluation of wellbeing interventions, the reports highlighted six key barriers to doing so; including lack of time, lack of appropriate baseline or benchmark data, and lack of appropriate evaluation criteria. ${ }^{20}$ Nottingham University with partners are currently working to develop a common set of criteria for evaluating digital mental health tools, such as mobile apps and websites. $^{21}$

By using the information gained from this survey regarding what support HCPs want, those developing services can incorporate coproduction in its design. Co-production is not a new concept, but it is one with renewed prominence and reach in contemporary policy discourse. It refers to joint working between people or groups who have traditionally been separated into categories of user and producer.

The National Endowment for Science, Technology and the Arts (NESTA13) argue that, where activities are co-produced, services become far more effective agents of change. ${ }^{22}$

Some of the support that HCPs wanted can be addressed with simple, low-cost interventions eg setting up virtual peer-support groups. Some require a culture shift within workplaces, for 
example, HCPs asked for supportive and visible managers. Others, including cultural, faith-based and tailored support, require an understanding of local HCPs' demographics and support networks. Adequate PPE requires a national procurement strategy as well as local pathways. Addressing the breadth of support needs that have been identified in this survey requires a multi-faceted, collaborative and joined-up local, regional and national approach.

\section{Strengths and limitations}

We had responses from a wide variety of HCPs and were able to identify the causes of anxiety and what resources and support people wanted using a combination of direct questions and free text comments using a brief 2-minute survey that managed to capture a wide breadth of information.

There are some limitations to our findings. The pre-pandemic anxiety score was a self-rated score at the time of the pandemic and therefore would have been affected by recall bias. Selfperceived anxiety was measured on a Likert-type rating scale, however, prevalence of anxiety may have been different with the use of validated tools such as the GAD-7 scale.

Hospital nurses were the group who had the highest anxiety score, with a median anxiety score of 10 . However, as the numbers of nurse respondents were low, statistical analysis was limited. This highlights a clear need to explore further with a larger cohort as a matter of urgency.

This survey did not record the grade of doctors (eg grade of training). Studies from China highlight that middle-grade doctors' mental health was disproportionately negatively affected during the COVID-19 pandemic. ${ }^{14}$ Further study to investigate this in the UK is needed.

Generalisability of the findings may be an issue as many respondents were based in London, which has been harder hit. In particular, boroughs in the north and east areas of London have some of the highest morbidity and mortality from COVID-19 in the country. ${ }^{23}$ However, the causes of anxiety and the themes of support identified in this survey are very similar to other studies and the HCPs wellbeing interventions found to be effective in meta-analysis and rapid review. 8,24

Due to the aim of keeping the survey short, allied HCPs ( 80 respondents) were not further stratified as it was beyond the scope of the survey; as such, there could be differences within these disparate subgroups of HCPs and this could be assessed in future studies. The patient facing nature of roles were not specifically assessed, which could affect the risk of exposure to SARS-CoV-2. Further study here would be interesting as evidence suggests that non-medically trained HCPs experienced higher rates of psychological trauma from the COVID-19 pandemic than other HCPs. $^{25}$

\section{Conclusion}

Anxiety levels significantly increased in HCPs during the COVID-19 pandemic, with the highest increase in doctors and nurses. The most common reasons people identified as causing the most anxiety were related to concerns about exposure to SARS-CoV-2, lack of PPE and the lack of available SARS-CoV-2 testing.

Many HCPs felt that there was inadequate support for their mental health and wellbeing, with common themes about what support was deemed important. This information should be utilised for improving and developing co-produced, flexible and tailored services. To adequately address the breadth of support needs identified in this survey requires a multi-faceted and collaborative local, regional and national response.

\section{Summary}

\section{What is known?}

Studies from previous pandemics have shown adverse psychological impact on healthcare professionals (HCPs).

\section{What is the question?}

Has anxiety increased in HCPs in the UK during the COVID-19 pandemic? What are the specific causes and what support do HCPs want for their mental health and wellbeing?

\section{What was found?}

Perceived anxiety in HCPs has increased in the UK during the COVID-19 pandemic. Causes were wide ranging, but the main reasons were related to concerns about risk of exposure to SARSCoV-2 and lack of PPE. A large proportion of HCPs felt there was inadequate support and identified what support they wanted.

\section{What is the implication for practice now?}

Strategic interventions to address the anxieties of HCPs are required as matter of urgency. A multi-faceted and collaborative local, regional and national approach is needed to address the breadth of support needs identified in this survey.

\section{Supplementary material}

Additional supplementary material may be found in the online version of this article at www.rcpjournals.org/clinmedicine: S1 - Full survey questionnaire.

S2 - Figures charting anxiety, across job role and job sector.

\section{Funding}

Survey tool costs were funded by Newham Together Training Hub. The funding body had no involvement in the design, analysis, writing or decision to submit this manuscript.

\section{References}

1 Office for National Statistics. Coronavirus and anxiety, Great Britain: 3 April 2020 to 10 May 2020. ONS, 2020. www.ons.gov. uk/peoplepopulationandcommunity/wellbeing/articles/coronavir usandanxietygreatbritain/3april2020to10may2020 [Accessed 13 September 2020].

2 Salari N, Hosseinian-Far A, Jalali R et al. Prevalence of stress, anxiety, depression among the general population during the COVID-19 pandemic: A systematic review and meta-analysis. Global Health 2020;16:57.

3 Lai J, Ma S, Wang $Y$ et al. Factors associated with mental health outcomes among health care workers exposed to coronavirus disease 2019. JAMA Netw Open 2020;3:e203976.

4 Shanafelt T, Ripp J, Trockel M. Understanding and addressing sources of anxiety among health care professionals during the COVID-19 pandemic. JAMA 2020;323:2133-4.

5 EMERGE. CERA: COVID-19 Emergency Response Assessment: a professional survey. EMERGE, 2020. www.emergeresearch.org/trial/ cera [Accessed 05 September 2020].

6 Queen Mary University of London. COVID19 and physical and emotional wellbeing of HCP (COPE-HCP). ClinicalTrials.gov, 2020. 
https://clinicaltrials.gov/ct2/show/NCT04433260?term=ajay+gupta \&cond=covid-19+healthcare+professionals\&draw $=2 \&$ rank $=1$ [Accessed 13 September 2020].

7 Stuijfzand S, Deforges C, Sandoz V et al. Psychological impact of an epidemic/pandemic on the mental health of healthcare professionals: a rapid review. BMC Public Health 2020:20:1230.

8 Gold JA. Covid-19: Adverse mental health outcomes for healthcare workers. BMJ 2020;369:m1815.

9 Pearman A, Hughes ML, Smith EL, Neupert SD. Mental health challenges of United States healthcare professionals during COVID-19. Front Psychol 2020;11:2065.

10 Lester D, Hvezda J, Sullivan S, Plourde R. Maslow's hierarchy of needs and psychological health. J Gen Psychol 1983;109:83-5.

11 Sharma M, Yadav K, Yadav N, Ferdinand KC. Zika virus pandemicanalysis of Facebook as a social media health information platform. Am J Infect Control 2017:45:301-2.

12 Kisely S, Warren N, McMahon L et al. Occurrence, prevention, and management of the psychological effects of emerging virus outbreaks on healthcare workers: rapid review and meta-analysis. BMJ 2020;369:m1642.

13 Lopresti AL, Hood SD, Drummond PD. A review of lifestyle factors that contribute to important pathways associated with major depression: Diet, sleep and exercise. J Affect Disord 2013;148: $12-27$.

14 Corley A, Hammond NE, Fraser JF. The experiences of health care workers employed in an Australian intensive care unit during the H1N1 Influenza pandemic of 2009: A phenomenological study. Int J Nurs Stud 2010;47:577-85.

15 NHS Practitioner Health. Practitioner Health. NHS. www.practitionerhealth.nhs.uk [Accessed 13 September 2020].

16 Healthy London Partnership. Good Thinking digital wellbeing service. Healthy London Partnership. www.healthylondon.org/goodthinking-digital-wellbeing [Accessed 13 September 2020].

17 Our NHS People. Wellbeing apps. NHS. https://people.nhs.uk/help/ support-apps [Accessed 13 September 2020].
18 Blake H, Bermingham F. Psychological Wellbeing for Health and Care Workers. Nottingham University, 2020. www.nottingham. ac.uk/toolkits/play_22794\#resume=1 [Accessed 05 September 2020].

19 City, University of London. COVID-AAD study. City, University of London, 2020. https://cityunilondon.eu.qualtrics.com/jfe/form/ SV_3JVEjtwvOS44BBb [Accessed 05 September 2020].

20 NHS Employers. Developing your health and wellbeing strategy. NHS. www.nhsemployers.org/retention-and-staff-experience/ health-and-wellbeing/developing-your-health-and-wellbeingstrategy [Accessed 06 September 2020].

21 University of Nottingham. Digital mental health and wellbeing. University of Nottingham. www.nottingham.ac.uk/research/ groups/digitalmentalhealthandwellbeing/index.aspx [Accessed 06 September 2020].

22 Boyle D, Harris M. The challenge of co-production: How equal partnerships between professionals and the public are crucial to improving public services. London: NESTA, 2009.

23 Mohdin A. 'Every day I hear about a Covid-19 death': life in the UK's worst-affected area. Guardian 01 May2020.

24 Shanafelt T, Ripp ], Trockel M. Understanding and addressing sources of anxiety among health care professionals during the COVID-19 pandemic. JAMA 2020;323:2133-4.

25 Tan BYQ, Chew NWS, Lee GKH et al. Psychological impact of the COVID-19 pandemic on health care workers in Singapore. Ann Intern Med. 2020:M20-1083.

Address for correspondence: Dr Imrana Siddiqui,

Woodgrange Medical Practice, 40-42 Woodgrange Road, London E7 0QH, UK.

Email: imranasiddiqui@nhs.net 